\title{
A NEW FOURTH-ORDER FINITE-DIFFERENCE METHOD FOR SOLVING DISCRETE-ORDINATES SLAB TRANSPORT EQUATIONS*
}

\author{
BENY NETA $\dagger$ AND H. D. VICTORY, JR. $\dagger$
}

\begin{abstract}
This work is concerned with a theoretical study of a new fourth-order finite-difference scheme for spatially discretizing the discrete-ordinates equations for solving numerically the slab transport (Boltzmann) equation. This analysis considers the quadratic continuous method, whose derivation parallels that of the commonly used diamond difference and linear discontinuous schemes from balance equations for particle conservation across a spatial cell. We provide a convergence analysis of this method and prove that superconvergence phenomena are present for cell-edge and cell-average fluxes. We also present results from an $S_{2}$-test problem to show that the asymptotic convergence rates are observed on rather coarse spatial meshes.
\end{abstract}

1. Introduction. Several recent articles [1], [3]-[10] have considered various finite-difference approximations to the discrete-ordinates equations for linear transport in slab geometry. In particular, Larsen and Miller [5], and Lee and Vaidyanathan [7], have obtained estimates of the orders of the discretization errors for several of these methods as applied to transport along a fixed direction without scattering. Many of the results in these works were extended by Larsen and Nelson [6] who showed that the orders of discretization errors obtained in the aforementioned works remain valid for the monoenergetic, discrete-ordinates approximation with scattering. The specific difference methods considered by Larsen and Nelson are the step characteristic, diamond difference, linear discontinuous, linear characteristic, linear moments, and quadratic methods described in [1], [5]. One of the salient results in the work by Larsen and Nelson is that the discretization errors for the cell-edge and cell-average flux approximates to the linear characteristic and quadratic methods of Gopinath et al., and of Vaidyanathan [4], [9], [10], are of order four, whereas the discretization errors for the corresponding approximates to the widely used linear discontinuous method are of order three. These theoretical results had been confirmed numerically by Larsen and Miller in [5], who found that the asymptotic convergence rates for the cell-edge and cell-average fluxes to the linear characteristic and quadratic methods can be observed on coarser meshes than for the lesser rate of order three for the corresponding fluxes of the linear discontinuous method. For these reasons, the linear characteristic method was selected in [5] to be the most preferable method for effecting slab geometry transport calculations.

It is well known that both the widely used diamond difference and linear discontinuous methods can be adapted to effect calculations in more complicated, onedimensional transport media than planar (e.g., in one-dimensional spherical and cylindrical geometries). The question is certainly begged then of generalizing the "balance equation approach" yielding these two methods in order to obtain more accurate methods. One such method considered in this paper is the quadratic continuous method suggested to the second author by E. Larsen. A quadratic approximation to the discrete-ordinates angular flux is constructed over a spatial mesh from the known entering flux along with the exciting flux and the cell-averaged flux obtained from the

* Received by the editors December 1, 1981, and in revised form March 16, 1982. This research was supported by The Center for Energy Research of Texas Tech University, and by the U.S. National Science Foundation under grant CPE 8007396. Support for the graduate research assistants involved in this work was provided by a grant from the Institute for University Research-Arts and Sciences, Texas Tech University.

$\dagger$ Institute for Numerical Transport Theory, Department of Mathematics, Texas Tech University, Lubbock, Texas 79409. 
two balance equations. We shall see in our analysis that the discretization errors for the cell-edge and cell-average fluxes are one order higher than the third-order discretization errors for these flux quantities computed by the widely used linear discontinuous method. Also, we point out that the storage requirements of the quadratic continuous method are similar to those of the linear discontinuous method. These considerations have prompted us to deem the quadratic continuous method worthy of theoretical and numerical study.

Although the orientation of this work is analytical, we shall address some of the potential problems which arise in implementing the quadratic continuous method computationally. One such problem will be to devise a technique to make this method "positive" so that nonnegative flux approximations are computed from nonnegative source data. Also, we perform preliminary numerical experiments with a sample benchmark problem [5, p. 81] to see whether the theoretically derived asymptotic rates of convergence can be observed on relatively coarse meshes. In a separate paper, we shall report the results from computer studies using other benchmark problems [1]. These compare, for the linear characteristic method, the quadratic continuous method, and some schemes of order six, the computer times required to complete one inner iteration and the storage requirements needed to obtain an approximation to the cell-edge and cell-average fluxes to within a given accuracy.

We refer the reader to $[6, \S 2]$ for a description of the assumptions and notation for the total and scattering cross section data, of the appropriate function spaces and norms used, and of the definition of the various spatial Legendre moments. Further, we recall that a family of spatial nets is quasiuniform if there exists a constant $C$ such that $h \leqq C \min \left\{h_{j}: 1 \leqq j \leqq J\right\}$ for all nets in the family, where $h_{j}$ is the width of a spatial cell $C_{j}=\left[x_{j-1 / 2}, x_{j+1 / 2}\right]$, and $h=\max \left\{h_{j}: 1 \leqq j \leqq J\right\}$. We do wish to point out at the outset that the total cross section data, the scattering cross section data, and the ratio of scattered particles to colliding ones-denoted in the ensuing sections by $\sigma, k$, and $c$ respectively-will be assumed piecewise constant in $x \in[0, a]$, where $a$ is the thickness of the slab. Also, the variable $\mu$ will indicate the cosine of the angle of particle direction, with $\left\{\mu_{i}: 1 \leqq i \leqq N\right\}$ denoting the discrete quadrature nodes taken in the discrete-ordinates formulation. At this point, we define the zeroth and first spatial moments of a component $f_{i}(i=1, \cdots N)$ of $f \in C_{p}^{N}[0, a]$ over the internal $C_{j}$ by

$$
\begin{gathered}
\left(\mathbf{M}_{0} f\right)_{i, j}=\frac{1}{h_{j}} \int_{x_{j-1 / 2}}^{x_{j+1 / 2}} f_{i}(s) d s, \\
\left(\mathbf{R}_{1} f\right)_{i, j}=\frac{6}{h_{j}^{2}} \int_{x_{j-1 / 2}}^{x_{j+1 / 2}}\left(s-x_{j}\right) f_{i}(s) d s .
\end{gathered}
$$

We shall also use the quantity $\left(\overline{\mathbf{R}}_{1} f\right)_{i, j}$, defined for any $f \in C_{p}^{N}[0, a]$, by

$$
\left(\overline{\mathbf{R}}_{1} f\right)_{i, j}=\frac{f_{i}\left(x_{j+1 / 2}\right)-f_{i}\left(x_{j-1 / 2}\right)}{2} .
$$

If, moreover, $f$ has piecewise continuous third derivatives, then $\left(\overline{\mathbf{R}}_{1} f\right)_{i, j}$ approximates $\left(\mathbf{R}_{1} f\right)_{i, j}$ to within $O\left(h_{j}^{3}\right)$, and both $\overline{\mathbf{R}}_{1}$ and $\mathbf{R}_{1}$ agree on quadratic polynomials defined on $C_{j}$ for each $j$.

In the following section, we shall study the convergence properties of the quadratic continuous method. Our concluding section will summarize the results of preliminary numerical experiments on an $S_{2}$ (two angular quadrature nodes), monoenergetic test problem [5, p. 81]. 


\section{Analysis of the quadratic continuous method}

2.1. Global error estimates. The convergence analysis requires some effort, primarily because of the quadratic function approximating $\psi_{i}(x), i=1,2, \cdots, N$, for $x \in C_{j}=\left[x_{j-1 / 2}, x_{j+1 / 2}\right]$. We recall that in the diamond difference scheme [1], [5], the assumption of linearity of $\psi_{i}(x), x \in C_{j}$, enables only one balance equation to be solved for the right-edge flux $\psi_{i}\left(x_{j+1 / 2}\right), \mu_{i}>0\left(\psi_{i}\left(x_{j-1 / 2}\right), \mu_{i}<0\right)$ in terms of the internal sources and $\psi_{i}\left(x_{j-1 / 2}\right)$ (respectively, $\psi_{i}\left(x_{j+1 / 2}\right)$ ), since the zeroth spatial moment of $\psi_{i}$ then becomes known. Analogously, in the quadratic continuous method, the assumption of a quadratic behavior of $\psi_{i}(x)$ over each $C_{j}$ enables us to utilize two balance equations to be solved for the right-edge flux $\left(\mu_{i}>0\right)$ or the left-edge flux $\left(\mu_{i}<0\right)$, and the zeroth spatial moment of $\psi_{i}$ on $C_{i}$ in terms of the internal sources and corresponding (known) edge fluxes - the first spatial moment of $\psi_{i}$ having been known when $\psi_{i}$ is assumed quadratic over $C_{j}$.

The fundamental equation, whose solution we approximate, is

$$
\begin{aligned}
\mu_{i} \frac{d \psi_{i}(x)}{d x}+\sigma(x) \psi_{i}(x) & =\sigma(x)\left[\mathbf{M}_{L C}(\mathbf{S} \psi+\bar{q})\right]_{i}(x), \quad x \in C_{j}, \\
\psi_{i}(0) & =0, \quad \mu_{i}>0, \\
\psi_{i}(a) & =0, \quad \mu_{i}<0,
\end{aligned}
$$

with

$$
\left(\mathbf{M}_{L C} f\right)_{i}(x)=\left(\mathbf{M}_{0} f\right)_{i, j}+\frac{2\left(x-x_{j}\right)}{h_{j}}\left(\overline{\mathbf{R}}_{1} f\right)_{i, j}, \quad x \in \stackrel{C}{C_{j}},
$$

where $\dot{C}_{j}$ is the interior of the cell $C_{j}$. Equation (2.1a) leads to two spatial Legendre moment equations for $\psi_{i}\left(x_{j+1 / 2}\right)$ and $\left(\mathbf{M}_{0} \psi\right)_{i, j}$, with $\mu_{i}>0$ and $\varepsilon_{j i}=\sigma\left(x_{j}\right) h_{j} / \mu_{i}$ :

$$
\begin{gathered}
\frac{1}{\varepsilon_{j i}}\left(\psi_{i}\left(x_{j+1 / 2}\right)-\psi_{i}\left(x_{j-1 / 2}\right)\right)+\left(\mathbf{M}_{0} \psi\right)_{i, j}=\left[\mathbf{M}_{0}(\mathbf{S} \psi+\bar{q})\right]_{i, j} \\
\frac{3}{\varepsilon_{j i}}\left(\psi_{i}\left(x_{j+1 / 2}\right)+\psi_{i}\left(x_{j-1 / 2}\right)-2\left(\mathbf{M}_{0} \psi\right)_{i, j}\right)+\left[\frac{\psi_{i}\left(x_{j+1 / 2}\right)-\psi_{i}\left(x_{j-1 / 2}\right)}{2}\right]=\left[\overline{\mathbf{R}}_{1}(\mathbf{S} \psi+\bar{q})\right]_{i, j}
\end{gathered}
$$

Solving for $\left(\mathbf{M}_{0} \psi\right)_{i, j}$ and $\psi_{i}\left(x_{j+1 / 2}\right)$ when $\mu_{i}>0$ (or $\psi_{i}\left(x_{j-1 / 2}\right)$ when $\mu_{i}<0$ ), and treating the other quantities as known, yields

$$
\psi_{i}\left(x_{j+\nu / 2}\right)=\left[\frac{12-6\left|\varepsilon_{j i}\right|+\varepsilon_{j i}^{2}}{12+6\left|\varepsilon_{j i}\right|+\varepsilon_{j i}^{2}}\right] \psi_{i}\left(x_{j-\nu / 2}\right)+\left[\frac{12\left|\varepsilon_{j i}\right|}{12+6\left|\varepsilon_{j i}\right|+\varepsilon_{j i}^{2}}\right]\left[\mathbf{M}_{0}(\mathbf{S} \psi+\bar{q})\right]_{i, j}
$$

$$
\begin{gathered}
+\nu\left[\frac{2 \varepsilon_{j i}^{2}}{12+6\left|\varepsilon_{j i}\right|+\varepsilon_{j i}^{2}}\right]\left[\overline{\mathbf{R}}_{1}(\mathbf{S} \psi+\bar{q})\right]_{i, j}, \\
\left(\mathbf{M}_{0} \psi\right)_{i, j}=\left[\frac{12}{12+6\left|\varepsilon_{j i}\right|+\varepsilon_{j i}^{2}}\right] \psi_{i}\left(x_{j-\nu / 2}\right)+\left[\frac{6\left|\varepsilon_{j i}\right|+\varepsilon_{j i}^{2}}{12+6\left|\varepsilon_{j i}\right|+\varepsilon_{j i}^{2}}\right]\left[\mathbf{M}_{0}(\mathbf{S} \psi+\bar{q})\right]_{i, j} \\
-\nu\left[\frac{2\left|\varepsilon_{j i}\right|}{12+6\left|\varepsilon_{j i}\right|+\varepsilon_{j i}^{2}}\right]\left[\overline{\mathbf{R}}_{1}(\mathbf{S} \psi+\bar{q})\right]_{i, j},
\end{gathered}
$$


where $\nu$ is the sign of $\mu_{i}$. Our quadratic interpolant which yields $\psi_{i}\left(x_{j-1 / 2}\right), \psi_{i}\left(x_{j+1 / 2}\right)$, and $\left(\mathbf{M}_{0} \psi\right)_{i, j}$ is, for $x \in C_{j}$,

$$
\begin{aligned}
\psi_{i}(x)= & \frac{1}{2}\left\{\frac{24-\varepsilon_{j i}^{2}-24 \nu\left|\varepsilon_{j i}\right|\left(\frac{x-x_{j}}{h_{j}}\right)+12 \varepsilon_{j i}^{2}\left(\frac{x-x_{j}}{h_{j}}\right)^{2}}{12+6\left|\varepsilon_{j i}\right|+\varepsilon_{j i}^{2}}\right\} \psi_{i}\left(x_{j-\nu / 2}\right) \\
& +\frac{3}{2}\left\{\frac{4\left|\varepsilon_{j i}\right|+\varepsilon_{j i}^{2}+8 \nu\left|\varepsilon_{j i}\right|\left(\frac{x-x_{j}}{h_{j}}\right)-4 \varepsilon_{j i}^{2}\left(\frac{x-x_{j}}{h_{j}}\right)^{2}}{12+6\left|\varepsilon_{j i}\right|+\varepsilon_{j i}^{2}}\right\}\left[\mathbf{M}_{0}(\mathbf{S} \psi+\bar{q})\right]_{i, j} \\
& +\frac{1}{2}\left\{\frac{-\nu\left[6\left|\varepsilon_{j i}\right|+\varepsilon_{j i}^{2}-\left(12 \varepsilon_{j i}^{2}+24\left|\varepsilon_{j i}\right|\right)\left(\frac{x-x_{j}}{h_{j}}\right)^{2}\right]+4 \varepsilon_{j i}^{2}\left(\frac{x-x_{j}}{h_{j}}\right)}{12+6\left|\varepsilon_{j i}\right|+\varepsilon_{j i}^{2}}\right\}\left[\overline{\mathbf{R}}_{1}(\mathbf{S} \psi+\bar{q})\right]_{i, j} .
\end{aligned}
$$

The quadratic continuous method can be expressed in the form $\psi=$ $\mathbf{L}_{Q C} \mathbf{M}_{L C}(\mathbf{S} \psi+\bar{q})$, with $\mathbf{L}_{Q C}$ defined for $x \in C_{j}$ and $\mu_{i}>0$ by

$\left(\mathbf{L}_{Q C} f\right)_{i}(x)=\frac{1}{2}\left\{\frac{24-\varepsilon_{j i}^{2}-24 \varepsilon_{j i}\left(\frac{x-x_{j}}{h_{j}}\right)+12 \varepsilon_{j i}^{2}\left(\frac{x-x_{j}}{h_{j}}\right)^{2}}{12+6 \varepsilon_{j i}+\varepsilon_{j i}^{2}}\right\}$

$$
\begin{array}{r}
\cdot \sum_{j^{\prime}=1}^{j-1}\left[\prod_{m=j^{\prime}+1}^{j-1} \frac{12-6 \varepsilon_{m i}+\varepsilon_{m i}^{2}}{12+6 \varepsilon_{m i}+\varepsilon_{m i}^{2}}\right] \frac{\varepsilon_{j^{\prime} i}}{12+6 \varepsilon_{j^{\prime} i}+\varepsilon_{j^{\prime} i}^{2}}\left[12\left(\mathbf{M}_{0} f\right)_{i, j^{\prime}}+2 \varepsilon_{j^{\prime} i}\left(\overline{\mathbf{R}}_{1} f\right)_{i, j^{\prime}}\right] \\
+\frac{\varepsilon_{j i}}{2\left(12+6 \varepsilon_{j i}+\varepsilon_{j i}^{2}\right)}\left\{\left[12+3 \varepsilon_{j i}+24\left(\frac{x-x_{j}}{h_{j}}\right)-12 \varepsilon_{j i}\left(\frac{x-x_{j}}{h_{j}}\right)^{2}\right]\left(\mathbf{M}_{0} f\right)_{i, j}\right. \\
\left.+\left[-6-\varepsilon_{j i}+4 \varepsilon_{j i}\left(\frac{x-x_{j}}{h_{j}}\right)+\left(12 \varepsilon_{j i}+24\right)\left(\frac{x-x_{j}}{h_{j}}\right)^{2}\right]\left(\overline{\mathbf{R}}_{1} f\right)_{i, j}\right\} .
\end{array}
$$

A similar formula can be constructed for the case $\mu_{i}<0$ by utilizing (2.5) in conjunction with (2.4). Our analysis will deal with the case $\mu_{i}>0$, as a similar analysis is possible for the case of negative $\mu_{i}$. We conclude from the preceding discussion that $\psi \in C^{N}[0, a]$.

LEMMA 1. The sequence of quadratic continuous approximations generated by a quasiuniform family of nets is consistent with the underlying discrete-ordinates problem,

$$
\begin{aligned}
\mu_{i} \frac{d \psi_{i}^{e}}{d x}(x)+\sigma(x) \psi_{i}^{e}(x) & =c(x) \sigma(x) \sum_{m=1}^{N} \omega_{m} k\left(x, \mu_{m}, \mu_{i}\right) \psi_{m}^{e}(x)+q_{i}(x), \\
& \psi_{i}^{e}(0)=0, \quad \mu_{i}>0, \\
& \psi_{i}^{e}(0)=0, \quad \mu_{i}<0,
\end{aligned}
$$

(with $\left\{\mu_{i}: 1 \leqq i \leqq N\right\}$ the weights associated with the quadrature nodes).

Proof. We shall ultimately prove that (in the sup-norm),

$$
\left\|\left(\mathbf{L}_{Q C} \mathbf{M}_{L C}-\mathbf{L}\right)\left(\mathbf{S} \psi^{e}+\bar{q}\right)\right\|=O\left(h^{3}\right)
$$

over any quasiuniform family of nets, where $\mathbf{S} \psi^{e}+\bar{q}$ is the right-hand side of (2.7a), with $\sigma(x), x \in C_{j}$, a common factor to both terms, and $\mathbf{L}$ is the operator defined for $f \in C_{p}^{N}[0, a]$ by

$$
(\mathbf{L} f)_{i}(x)=\frac{1}{\mu_{i}} \int_{0}^{x} \exp \left\{\frac{-1}{\mu_{i}} \int_{x^{\prime}}^{x} \sigma(s) d s\right\} f_{i}\left(x^{\prime}\right) d x^{\prime}, \quad x \in C_{j}, \quad \mu_{i}>0 .
$$


To the desired end of proving (2.8), we find appropriate estimates for $\left\|\left(\mathbf{L}_{Q C}-\mathbf{L}\right) \mathbf{M}_{L C}\left(\mathbf{S} \psi^{e}+\bar{q}\right)\right\|$ for $\mathbf{S} \psi^{e}+\bar{q}$ considered as an element in $\mathfrak{P}_{3} C_{p}^{N}[0, a]$ (see, for example, $[6, \S 2(\mathrm{~b})]$ for a description of this function space), and with $\mathbf{L}_{Q C}$ defined by (2.6). We shall produce our estimates by first observing that, for $f \in \mathfrak{P}_{3} C_{p}^{N}[0, a]$, $\left|\left(\mathbf{M}_{0} f\right)_{i, j}\right| \leqq\|f\|$ and $\left|\left(\overline{\mathbf{R}}_{1} f\right)_{i, j}\right|=O\left(h_{j}\right), \mathbf{L} \mathbf{M}_{L C} f$ can be expressed for $\mu_{i}>0, x \in C_{j}$,

$$
\begin{aligned}
\left(\mathbf{L M}_{L C} f\right)_{i}(x)= & \exp \left\{\frac{-\sigma_{j}\left(x-x_{j-1 / 2}\right)}{\mu_{i}}\right\}\left[\sum_{j^{\prime}=1}^{j-1} \prod_{m=j^{\prime}+1}^{j-1} \exp \left(-\varepsilon_{m i}\right)\right] \\
& \cdot\left\{\left(1-\exp \left(-\varepsilon_{j^{\prime} i}\right)\right)\left(\mathbf{M}_{0} f\right)_{i, j^{\prime}}\right. \\
& \left.+\left(\left(1+\exp \left(-\varepsilon_{j^{\prime} i}\right)\right)-2 \varepsilon_{j^{\prime} i}^{-1}\left(1-\exp \left(-\varepsilon_{j^{\prime} i}\right)\right)\right)\left(\overline{\mathbf{R}}_{1} f\right)_{i, j^{\prime}}\right\} \\
& +\left[1-\exp \left\{\frac{-\sigma_{j}\left(x-x_{j-1 / 2}\right)}{\mu_{i}}\right\}\right]\left(\mathbf{M}_{0} f\right)_{i, j} \\
& +\left[2\left(\frac{x-x_{j}}{h_{j}}\right)-2 \varepsilon_{j i}^{-1}+\left(1+2 \varepsilon_{j i}\right) \exp \left\{\frac{-\sigma_{j}\left(x-x_{j-1 / 2}\right)}{\mu_{i}}\right\}\right]\left(\overline{\mathbf{R}}_{1} f\right)_{i, j} .
\end{aligned}
$$

In comparing $\mathbf{L M}_{L C} f$ with $\mathbf{L}_{Q C} \mathbf{M}_{L C} f$ in (2.6), we make use of the following asymptotics:

$$
\begin{gathered}
\frac{1}{2}\left\{\frac{24-\varepsilon_{j i}^{2}-24 \varepsilon_{j i}\left(\frac{x-x_{j}}{h_{j}}\right)+12 \varepsilon_{j i}^{2}\left(\frac{x-x_{j}}{h_{j}}\right)^{2}}{12+6 \varepsilon_{j i}+\varepsilon_{j i}^{2}}\right\}=\exp \left\{\frac{-\sigma_{j}\left(x-x_{j-1 / 2}\right)}{\mu_{i}}\right\}+O\left(h_{j}^{3}\right), \\
\frac{12-6 \varepsilon_{m i}+\varepsilon_{m i}^{2}}{12+6 \varepsilon_{m i}+\varepsilon_{m i}^{2}}=\exp \left(-\varepsilon_{m i}\right)+O\left(h_{m}^{5}\right),
\end{gathered}
$$

$$
\begin{gathered}
\frac{12 \varepsilon_{j^{\prime} i}}{12+6 \varepsilon_{j^{\prime} i}+\varepsilon_{j^{\prime} i}^{2}}=1-\exp \left(-\varepsilon_{j^{\prime} i}\right)+O\left(h_{j^{\prime}}^{5}\right) \\
\varepsilon_{j i} \frac{\left[12+3 \varepsilon_{j i}+24\left(\frac{x-x_{i}}{h_{j}}\right)-12 \varepsilon_{j i}\left(\frac{x-x_{j}}{h_{j}}\right)^{2}\right]}{2\left(12+6 \varepsilon_{j i}+\varepsilon_{j i}^{2}\right)}=1-\exp \left\{\frac{-\sigma_{j}\left(x-x_{j-1 / 2}\right)}{\mu_{i}}\right\}+O\left(h_{j}^{3}\right)
\end{gathered}
$$

$$
\begin{gathered}
\frac{2 \varepsilon_{j^{\prime} i}^{2}}{12+6 \varepsilon_{j^{\prime} i}+\varepsilon_{j^{\prime} i}^{2}}=1+\exp \left(-\varepsilon_{j^{\prime} i}\right)-\frac{2}{\varepsilon_{j^{\prime} i}}\left(1-\exp \left(-\varepsilon_{j^{\prime} i}\right)\right)+O\left(h_{j^{\prime}}^{4}\right), \\
\frac{\varepsilon_{j i}\left[-6-\varepsilon_{j i}+4 \varepsilon_{j i}\left(\frac{x-x_{j}}{h_{j}}\right)+\left(12 \varepsilon_{j i}+24\right)\left(\frac{x-x_{j}}{h_{j}}\right)^{2}\right]}{2\left(12+6 \varepsilon_{j i}+\varepsilon_{j i}^{2}\right)} \\
=2\left(\frac{x-x_{j}}{h_{j}}\right)-\frac{2}{\varepsilon_{j i}}+\left(1+\frac{2}{\varepsilon_{j i}}\right) \exp \left\{\frac{-\sigma_{j}\left(x-x_{j-1 / 2}\right)}{\mu_{i}}\right\}+O\left(h_{j}^{2}\right) .
\end{gathered}
$$

From (2.10) and (2.11), we can deduce that

$$
\left.\|\left(\mathbf{L}_{Q C}-\mathbf{L}\right) \mathbf{M}_{L C}\right)\left(\mathbf{S} \psi^{e}+\bar{q}\right) \|=O\left(h^{3}\right)
$$

as $h \rightarrow 0$ through any quasiuniform family of nets, since $\mathbf{S} \psi^{e}+\bar{q} \in \mathfrak{B}_{n} C_{p}^{N}[0, a]$ for arbitrary $n \geqq 0$, because the data in (2.7a) are piecewise constant. Finally, equation (2.8) will follow upon appeal to [6, Thm. 3], which shows that

$$
\left\|\mathbf{L}\left(\mathbf{I}-\mathbf{M}_{L C}\right) f\right\|=O\left(h^{3}\right), \quad f \in \mathfrak{P}_{3} C_{p}^{N}[0, a]
$$

as $h \rightarrow 0$ through any quasiuniform family of nets. Q.E.D. 
LEMMA 2. The global discretization errors for the quadratic continuous approximates,

$$
\left\|\psi-\psi^{e}\right\|=\max _{1 \leqq i \leqq N[0, a]} \sup _{1}\left|\psi_{i}(x)-\psi_{i}^{e}(x)\right|
$$

are $O\left(h^{3}\right)$ as $h \rightarrow 0$ through any quasiuniform family of nets.

Proof. It remains to prove the stability of the quadratic continuous approximations defined for each spatial cell $C_{j}$ by (2.5). Therefore, we must show that $\left(\mathbf{I}-\mathbf{L}_{Q C} \mathbf{M}_{L C} \mathbf{S}\right)^{-1}$ is uniformly bounded on $C_{p}^{N}[0, a]$ as $h \rightarrow 0$. Toward this end, we first observe that as operators on $C_{p}^{N}[0, a],\left|\left(\mathbf{M}_{0} f\right)_{i, j}\right| \leqq\|f\|,\left|\left(\overline{\mathbf{R}}_{1} f\right)_{i, j}\right| \leqq\|f\|$, and therefore, as a sequence of operators on $C_{p}^{N}[0, a],\left(\mathbf{L}_{Q C}-\mathbf{L}\right) \mathbf{M}_{L C}$ have norms $O\left(h^{2}\right)$ as $h \rightarrow 0$ over any quasiuniform family of nets. The collectively compact operator approximation theory of P. Anselone [2], applied in a manner similar to that of Larsen and Nelson in [6, Thm. 2], enables us to conclude $\mathbf{L} \mathbf{M}_{L C} \mathbf{S}$ is collectively compact, converging pointwise to $\mathbf{L S}$ on $C_{p}^{N}[0, a]$. We conclude that $\left\{\left(\mathbf{I}-\mathbf{L} \mathbf{M}_{L C} \mathbf{S}\right)^{-1}\right\}$ is uniformly bounded over any quasiuniform family of nets; and, by the Banach perturbation lemma [2, p. 3], that $\left\{\left(\mathbf{I}-\mathbf{L}_{Q C} \mathbf{M}_{L C} \mathbf{S}\right)^{-1}\right\}$ is likewise uniformly bounded. Along with (2.8), we conclude that the global discretization error for the quadratic continuous method,

$$
\left\|\psi-\psi^{e}\right\| \leqq\left\|\left(\mathbf{I}-\mathbf{L}_{Q C} \mathbf{M}_{L C} \mathbf{S}\right)^{-1}\right\|\left\|\left(\mathbf{L}_{Q C} \mathbf{M}_{L C}-\mathbf{L}\right)\left(\mathbf{S} \psi^{e}+\bar{q}\right)\right\|
$$

is $O\left(h^{3}\right)$ as $h \rightarrow 0$ through any quasiuniform family of nets. This completes the proof.

2.2. Superconvergence results. The superconvergence of the cell-average fluxes, and ultimately of the cell-edge fluxes, is a consequence of the following lemmas:

LEMMA 3. The sequence of operators $\left\{\left(\mathbf{I}-\mathbf{M}_{0} \mathbf{L}_{Q C} \mathbf{S}\right)^{-1}\right\}$ is uniformly bounded as $h \rightarrow 0$ through any quasiuniform sequence of nets.

LEMMA 4. The following estimates,

$$
\begin{gathered}
\left\|\mathbf{M}_{0} \mathbf{L}_{Q C} \mathbf{M}_{1} \mathbf{S}\left(\psi-\psi^{e}\right)\right\|=O\left(h^{4}\right), \\
\left\|\mathbf{M}_{0}\left(\mathbf{L}_{Q C} \mathbf{M}_{L C}-\mathbf{L}\right)\left(\mathbf{S} \psi^{e}+\bar{q}\right)\right\|=O\left(h^{4}\right),
\end{gathered}
$$

are valid as $h \rightarrow 0$ through any quasiuniform family of nets, where $\mathbf{M}_{1}$ is defined for $f \in C_{p}^{N}[0, a]$ by

$$
\left(\mathbf{M}_{1} f\right)_{i}(x)=\frac{2\left(x-x_{j}\right)}{h_{j}}\left\{\frac{f_{i}\left(x_{j+1 / 2}\right)-f_{i}\left(x_{j-1 / 2}\right)}{2}\right\}, \quad x \in \mathcal{C}_{j}
$$

Remarks. As discussed in $[6, \S 4]$, Lemmas 3 and 4 together will prove the superconvergence of the cell-average fluxes, i.e., that

$$
\left\|\mathbf{M}_{0}\left(\psi-\psi^{e}\right)\right\|=O\left(h^{4}\right)
$$

as $h \rightarrow 0$ through any quasiuniform family of nets. We conclude that a similar property is valid for the cell-edge fluxes:

$$
\max \left\{\left|\psi_{i}\left(x_{j+1 / 2}\right)-\psi_{i}^{e}\left(x_{j+1 / 2}\right)\right|: i=1,2, \cdots, N ; j=1,2, \cdots, J\right\}=O\left(h^{4}\right),
$$

as $h \rightarrow 0$, because of the facts that both $\psi_{i}$ and $\psi_{i}^{e}$ satisfy (2.3a), and that the exact boundary conditions $(2.7 \mathrm{~b}, \mathrm{c})$ are required of the approximates.

Proof of Lemma 3. It is straightforward to show that the operators $\left\{\left(\mathbf{L}_{Q C}-\mathbf{L}\right) \mathbf{M}_{0}\right\}$, considered on $C_{p}^{N}[0, a]$, have norms $\left\|\left(\mathbf{L}_{Q C}-\mathbf{L}\right) \mathbf{M}_{0}\right\|=O\left(h^{3}\right)$ as $h \rightarrow 0$ through any quasiuniform family of nets. By applying the Banach perturbation lemma, we conclude that $\left\{\left(\mathbf{I}-\mathbf{L}_{Q C} \mathbf{M}_{0} \mathbf{S}\right)^{-1}\right\}$ is a uniformly bounded sequence of operators, since 
$\left\{\left(\mathbf{I}-\mathbf{L} \mathbf{M}_{0} \mathbf{S}\right)^{-1}\right\}$ is [6, Thm. 2]. Finally, arguments as employed in [6, Thm. 5] yield the conclusion of the lemma.

Proof of Lemma 4. We observe that $\left(\mathbf{L}_{Q C} \mathbf{M}_{1} f\right)_{i}(x), x \in C_{j}$, is provided by (2.10) with $\left(\mathbf{M}_{0} f\right)_{i, j^{\prime}}=0,1 \leqq j^{\prime} \leqq j$. We can also deduce that $\left\|\mathbf{L}_{Q C} \mathbf{M}_{1}\right\|=O(h)$ as $h \rightarrow 0$ through any quasiuniform family of nets, where the norm here is taken to be the operator norm. Such a conclusion follows from the fact that

$$
\begin{aligned}
\frac{\varepsilon_{j i}}{2\left(12+6 \varepsilon_{j i}+\varepsilon_{j i}^{2}\right)}\left[-6-\varepsilon_{j i}+4 \varepsilon_{j i}\left(\frac{x-x_{j}}{h_{j}}\right)+\left(12 \varepsilon_{j i}+24\right)\left(\frac{x-x_{j}}{h_{j}}\right)^{2}\right] \\
=\frac{2\left(x-x_{j}\right)}{h_{j}}-\frac{2}{\varepsilon_{j i}}+\left(1+\frac{2}{\varepsilon_{j i}}\right) \exp \left\{\frac{-\sigma_{j}\left(x-x_{j-1 / 2}\right)}{\mu_{i}}\right\}+O\left(h_{j}^{2}\right)=O(h) .
\end{aligned}
$$

Equation (2.16a) is easily established.

For establishing (2.16b), we observe that, with $g=\mathbf{S} \psi^{e}+\bar{q},\left\|\mathbf{M}_{0}\left(\mathbf{L}_{Q C} \mathbf{M}_{L C}-\mathbf{L}\right) g\right\| \leqq$ $\left\|\mathbf{M}_{0}\left(\mathbf{L}_{Q C}-\mathbf{L}\right) \mathbf{M}_{L C} g\right\|+\left\|\mathbf{M}_{0} \mathbf{L}\left(\mathbf{M}_{L C}-\mathbf{I}\right) g\right\|$. Since $\left\|\mathbf{M}_{0} \mathbf{L}\left(\mathbf{M}_{L C}-\mathbf{I}\right) g\right\|=O\left(h^{4}\right)$ was obtained in $[6, \S 4(b)]$ for $g \in \mathfrak{P}_{3} C_{p}^{N}[0, a]$, we only need to show that $\left\|\mathbf{M}_{0}\left(\mathbf{L}_{Q C}-\mathbf{L}\right) \mathbf{M}_{L C} g\right\|=$ $O\left(h^{4}\right)$ as $h \rightarrow 0$. Toward this end, we compute

$$
\begin{aligned}
\left(\mathbf{M}_{0} \mathbf{L}_{Q C} \mathbf{M}_{L C} g\right)_{i, j}= & {\left[\frac{12}{12+6 \varepsilon_{j i}+\varepsilon_{j i}^{2}}\right]_{j^{\prime}=1}^{j-1} \prod_{m=j^{\prime}+1}^{j-1}\left[\frac{12-6 \varepsilon_{m i}+\varepsilon_{m i}^{2}}{12+6 \varepsilon_{m i}+\varepsilon_{m i}^{2}}\right] } \\
& \cdot \frac{\varepsilon_{j^{\prime} i}}{12+6 \varepsilon_{j^{\prime} i}+\varepsilon_{j^{\prime} i}^{2}}\left[12\left(\mathbf{M}_{0} g\right)_{i, j^{\prime}}+2 \varepsilon_{j^{\prime} i}\left(\overline{\mathbf{R}}_{1} g\right)_{i, j^{\prime}}\right] \\
& +\left[\frac{6 \varepsilon_{j i}+\varepsilon_{j i}^{2}}{12+6 \varepsilon_{j i}+\varepsilon_{j i}^{2}}\right]\left(\mathbf{M}_{0} g\right)_{i, j}-\left[\frac{2 \varepsilon_{j i}}{12+6 \varepsilon_{j i}+\varepsilon_{j i}^{2}}\right]\left(\overline{\mathbf{R}}_{1} g\right)_{i, j .} .
\end{aligned}
$$

We get a corresponding expression for $\left(\mathbf{M}_{0} \mathbf{L} \mathbf{M}_{L C} g\right)_{i, j}$ by replacing the rational expressions in the summation by the exponential terms in the asymptotic expressions in (2.11), and by exponential terms in the following:

$$
\begin{gathered}
\frac{1-\exp \left(-\varepsilon_{j i}\right)}{\varepsilon_{j i}}=\frac{12}{12+6 \varepsilon_{j i}+\varepsilon_{j i}^{2}}+O\left(h_{j}^{4}\right), \\
1-\frac{1}{\varepsilon_{j i}}\left(1-\exp \left(-\varepsilon_{j i}\right)\right)=\frac{6 \varepsilon_{j i}+\varepsilon_{j i}^{2}}{12+6 \varepsilon_{j i}+\varepsilon_{j i}^{2}}+O\left(h_{j}^{4}\right), \\
{\left[\frac{-1}{\varepsilon_{j i}}+\frac{2}{\varepsilon_{j i}^{2}}-\exp \left(-\varepsilon_{j i}\right)\left(\frac{1}{\varepsilon_{j i}}+\frac{2}{\varepsilon_{j i}^{2}}\right)\right]=\frac{-2 \varepsilon_{j i}}{12+6 \varepsilon_{j i}+\varepsilon_{j i}^{2}}+O\left(h_{j}^{3}\right) .}
\end{gathered}
$$

After comparing the terms in $\left(\mathbf{M}_{0} \mathbf{L}_{Q C} \mathbf{M}_{L C} g\right)_{i, j}$ with corresponding terms in $\left(\mathbf{M}_{0} \mathbf{L} \mathbf{M}_{L C} g\right)_{i, j}$, while using the fact that $\left|\left(\overline{\mathbf{R}}_{1} g\right)_{i, j}\right|=O(h)$, with $g \in \mathfrak{P}_{3} C_{p}^{N}[0, a]$, we get (2.16b). This completes the proof of Lemma 4.

Q.E.D.

2.3. Further convergence results. We summarize the results in the preceding two subsections as the following:

THEOREM. The global discretization errors for the quadratic continuous finitedifference approximations to the discrete-ordinates equations are $O\left(h^{3}\right)$ as $h \rightarrow 0$ through any quasiuniform family of nets. Moreover, the approximations to the cell-edge and cell-average angular fluxes are accurate to within $O\left(h^{4}\right)$ as $h \rightarrow 0$ through any quasiuniform family of nets.

The following corollary provides some indication of the points in a spatial cell $C_{j}$ at which the approximations $\left\{\psi_{i}(x): i=1,2, \cdots, N\right\}$ in (2.5) are accurate to within 
$O\left(h^{4}\right)$ (at worst) and of points in $C_{j}$ at which $\left\{\psi_{i}(x): i=1, \cdots, N\right\}$ are accurate to within $O\left(h^{3}\right)$ :

COROllary. The cell-midpoint approximations $\left\{\psi_{i}(x): i=1, \cdots, N ; j=1,2\right.$, $\cdots, J\}$ are accurate to within $O\left(h^{4}\right)$, i.e.,

$$
\max \left\{\left|\psi_{i}\left(x_{j}\right)-\psi_{i}^{e}\left(x_{j}\right)\right|: i=1,2, \cdots, N ; j=1, \cdots, J\right\}=O\left(h^{4}\right) .
$$

At worst, the approximations $\left\{\psi_{i}\left(x_{j \pm 1 / 4}\right)\right\}$, with $x_{j \pm 1 / 4}=x_{j} \pm h_{j} / 4$, are accurate to within $O\left(h^{3}\right)$.

Proof. Because $\mathbf{S} \psi^{e}+\bar{q}$ lies in $\mathfrak{B}_{n} C_{p}^{N}[0, a]$ for any $n \geqq 0$, we can write (see, for example, Larsen and Nelson $[6, \S 2 \mathrm{~b}])$, for $x \in C_{j}, \mu_{i}>0$,

$$
\begin{aligned}
\left(\mathbf{S} \psi^{e}+\bar{q}\right)_{i}(x)= & {\left[\mathbf{M}_{0}\left(\mathbf{S} \psi^{e}+\bar{q}\right)\right]_{i, j}+\frac{2\left(x-x_{j}\right)}{h_{j}}\left[\overline{\mathbf{R}}_{1}\left(\mathbf{S} \psi^{e}+\bar{q}\right)\right]_{i, j} } \\
& +\left\{\frac{6}{h_{j}^{2}}\left(x-x_{j}\right)^{2}-\frac{1}{2}\right\}\left[\mathbf{R}_{2}\left(\mathbf{S} \psi^{e}+\bar{q}\right)\right]_{i, j}+O\left(h_{j}^{3}\right),
\end{aligned}
$$

with $\left[\mathbf{R}_{2}\left(\mathbf{S} \psi^{e}+\bar{q}\right)\right]_{i, j}$, the second spatial Legendre moment of the source terms defined for an arbitrary $f \in C_{p}^{N}[0, a]$ by

$$
\left(\mathbf{R}_{2} f\right)_{i, j}=\frac{5}{h_{j}} \int_{C_{j}} f_{i}(x)\left[\frac{6}{h_{j}^{2}}\left(x-x_{j}\right)^{2}-\frac{1}{2}\right] d x .
$$

Using (2.5), (2.7a), and (2.24), we compute and find

$$
\begin{aligned}
\psi_{i}^{e}\left(x_{j}\right) & -\psi_{i}\left(x_{j}\right) \\
= & \psi_{i}^{e}\left(x_{j-1 / 2}\right)\left[\exp \left(\frac{-\varepsilon_{j i}}{2}\right)-\frac{24-\varepsilon_{j i}^{2}}{24+12 \varepsilon_{j i}+2 \varepsilon_{j i}^{2}}\right] \\
& +\left[\mathbf{M}_{0}\left(\mathbf{S} \psi^{e}+\bar{q}\right)\right]_{i, j}\left[1-\exp \left(\frac{-\varepsilon_{j i}}{2}\right)-\left(\frac{12 \varepsilon_{j i}+3 \varepsilon_{j i}^{2}}{24+12 \varepsilon_{j i}+2 \varepsilon_{j i}^{2}}\right)\right] \\
& +\left[\overline{\mathbf{R}}_{1}\left(\mathbf{S} \psi^{e}+\bar{q}\right)\right]_{i, j}\left[\exp \left(\frac{-\varepsilon_{j i}}{2}\right)-\frac{2}{\varepsilon_{j i}}\left(1-\exp \left(\frac{-\varepsilon_{j i}}{2}\right)\right)+\frac{6 \varepsilon_{j i}+\varepsilon_{j i}^{2}}{24+12 \varepsilon_{j i}+2 \varepsilon_{j i}^{2}}\right] \\
& +\frac{24-\varepsilon_{j i}^{2}}{24+12 \varepsilon_{j i}+2 \varepsilon_{j i}^{2}}\left[\psi_{i}^{e}\left(x_{j-1 / 2}\right)-\psi_{i}\left(x_{j-1 / 2}\right)\right] \\
& +\frac{12 \varepsilon_{j i}+3 \varepsilon_{j i}^{2}}{24+12 \varepsilon_{j i}+2 \varepsilon_{j i}^{2}}\left[\mathbf{M}_{0} \mathbf{S}\left(\psi^{e}-\psi\right)\right]_{i, j} \\
& -\frac{6 \varepsilon_{j i}+\varepsilon_{j i}^{2}}{24+12 \varepsilon_{j i}+2 \varepsilon_{j i}^{2}}\left[\mathbf{R}_{1} \mathbf{S}\left(\psi^{e}-\psi\right)\right]_{i, j}+O\left(h_{j}^{4}\right),
\end{aligned}
$$

where the last $O\left(h_{i}^{4}\right)$ is due to the fact that $\left[\mathbf{R}_{2}\left(\mathbf{S} \psi^{e}+\bar{q}\right)\right]_{i, i}=O\left(h_{i}^{2}\right)$ with a coefficient also $O\left(h_{j}^{2}\right)$. It is easy to verify, by making use of the preceding theorem and noting that $\left[\overline{\mathbf{R}}_{1}\left(\mathbf{S} \psi^{e}+q\right)\right]_{i, j}=O(h)$, that each term in (2.26) is of $O\left(h_{j}^{4}\right)$. So the errors in the cell-midpoint approximations are (at worst) $O\left(h^{4}\right)$, and (2.23) follows.

For the interior points $x_{j \pm 1 / 4}=x_{j} \pm h_{j} / 4$, we compute

$$
\begin{aligned}
& \psi_{i}^{e}\left(x_{j-1 / 4}\right)-\psi_{i}\left(x_{j-1 / 4}\right) \\
& \quad=\psi_{i}^{e}\left(x_{j-1 / 2}\right)\left[\exp \left(\frac{-\varepsilon_{j i}}{4}\right)-\frac{96+24 \varepsilon_{j i}-\varepsilon_{j i}^{2}}{96+48 \varepsilon_{j i}+8 \varepsilon_{j i}^{2}}\right]
\end{aligned}
$$




$$
\begin{aligned}
& +\left[\mathbf{M}_{0}\left(\mathbf{S} \psi^{e}+\bar{q}\right)\right]_{i, j}\left[1-\exp \left(-\frac{\varepsilon_{j i}}{4}\right)-\frac{24 \varepsilon_{j i}+9 \varepsilon_{j i}^{2}}{96+48 \varepsilon_{j i}+8 \varepsilon_{j i}^{2}}\right] \\
& +\left[\overline{\mathbf{R}}_{1}\left(\mathbf{S} \psi^{e}+\bar{q}\right)\right]_{i, j}\left[-\frac{1}{2}+\exp \left(\frac{-\varepsilon_{j i}}{4}\right)-\frac{2}{\varepsilon_{j i}}\left(1-\exp \left(\frac{-\varepsilon_{j i}}{4}\right)\right)+\frac{18 \varepsilon_{j i}+5 \varepsilon_{j i}^{2}}{96+48 \varepsilon_{j i}+8 \varepsilon_{j i}^{2}}\right] \\
& +\frac{96+24 \varepsilon_{j i}-\varepsilon_{j i}^{2}}{96+48 \varepsilon_{j i}+8 \varepsilon_{j i}^{2}}\left[\psi_{i}^{e}\left(x_{j-1 / 2}\right)-\psi_{i}\left(x_{j-1 / 2}\right)\right]+\frac{24 \varepsilon_{j i}+9 \varepsilon_{j i}^{2}}{96+48 \varepsilon_{j i}+8 \varepsilon_{j i}^{2}}\left[\mathbf{M}_{0} \mathbf{S}\left(\psi^{e}-\psi\right)\right]_{i, j} \\
& +\frac{\left(-18 \varepsilon_{j i}-5 \varepsilon_{j i}^{2}\right)}{96+48 \varepsilon_{j i}+8 \varepsilon_{j i}^{2}}\left[\overline{\mathbf{R}}_{1} \mathbf{S}\left(\psi^{e}-\psi\right)\right]_{i, j}+O\left(h_{j}^{3}\right) .
\end{aligned}
$$

It is straightforward to show that each of the first three terms is $O\left(h_{j}^{3}\right)$, whereas each of the last three is $O\left(h_{j}^{4}\right)$. So, at worst, the errors in the approximations to $\psi_{i}^{e}\left(x_{j-1 / 4}\right)$,

$$
\max \left\{\left|\psi_{i}^{e}\left(x_{j-1 / 4}\right)-\psi_{i}\left(x_{j-1 / 4}\right)\right|: i=1,2, \cdots, N ; j=1,2, \cdots, J\right\},
$$

are $O\left(h_{j}^{3}\right)$ as $h \rightarrow 0$ through any quasiuniform family of nets. The analysis for $x_{j+1 / 4}$ is similar, and we omit the details. This completes the proof of the corollary.

Remark. The convergence analysis presented so far can be thought of as an error analysis for the result of one converged inner iteration in computing the angular flux for a particular energy group in the numerical treatment of multigroup, discreteordinates, slab transport problems. For the general multigroup problems, the dependent variable $\psi$ and the sources depend on the energy level $g_{0}$. The source terms consist of known internal source data for group $g_{0}$ as well as contributions from computed fluxes from other energy groups and are de facto known for one inner iteration sweep over the space-angle mesh. In this setting, the quadratic continuous scheme would be for $\mu_{i}>0$ :

$$
\begin{gathered}
\psi_{\mathrm{g}_{0}, i}\left(x_{j+1 / 2}\right)=f_{i, j, \mathrm{~g}_{0}} \psi_{\mathrm{g}_{0}, i}\left(x_{j-1 / 2}\right)+\left(1-f_{i, j, \mathrm{~g}_{0}}\right)\left[\mathbf{M}_{0} S_{\mathrm{g}_{0}}\right]_{i, j} \\
+2\left[1-\left(\frac{1}{2}+d_{i, j, \mathrm{~g}_{0}}\right)\left(1-f_{i, j, \mathrm{~g}_{0}}\right)\right]\left[\overline{\mathbf{R}}_{1} S_{\mathrm{g}_{0}}\right]_{i, j}, \\
{\left[\mathbf{M}_{0} \psi_{\mathrm{g}_{0}}\right]_{i, j}=\left[\mathbf{M}_{0} S_{\mathrm{g}_{0}}\right]_{i, j}-d_{i, j, \mathrm{~g}_{0}}\left[\psi_{\mathrm{g}_{0}, i}\left(x_{j+1 / 2}\right)-\psi_{\mathrm{g}_{0}, i}\left(x_{j-1 / 2}\right)\right],}
\end{gathered}
$$

with

$$
f_{i, j, \mathrm{~g}_{0}}=\frac{12-6 \varepsilon_{i, j, \mathrm{~g}_{0}}+\varepsilon_{i, j, \mathrm{~g}_{0}}^{2}}{12+6 \varepsilon_{i, j, \mathrm{~g}_{0}}+\varepsilon_{i, j, \mathrm{~g}_{0}}^{2}}, \quad d_{i, j, \mathrm{~g}_{0}}=\frac{1}{\varepsilon_{i, j, \mathrm{~g}_{0}}}, \quad \varepsilon_{i, j, \mathrm{~g}_{0}}=\frac{\sigma_{g_{0}}\left(x_{j}\right) h_{j}}{\mu_{i}} .
$$

The computed angular fluxes are folded into the running sum for the angular flux moments [1, pp. 113-114], which are then used to compute the zeroth and first spatial moment arrays, needed for the succeeding iteration sweep over the space-angle mesh.

The reader can easily deduce, by comparing $(2.28 \mathrm{a}-\mathrm{c})$ with similar expressions for the linear discontinuous and linear characteristic schemes [1, pp. 118 and 120 respectively], that the storage requirements for the quadratic continuous method for effecting one inner iteration sweep are precisely the same as those for the linear discontinuous and linear characteristic methods, discussed at length in [1] in terms of demands on the small core memory of a CDC-7600 computer.

3. Numerical results. In this section, we apply the quadratic continuous method to the following one-group test problem with two angular quadrature nodes selected:

$$
\begin{gathered}
\mu_{i} \frac{d}{d x} \psi_{i}(x)+\sigma \psi_{i}(x)=\sigma_{s} \sum_{m=1}^{2} \omega_{m} \psi_{m}(x)+q, \quad-1 \leqq x \leqq 1, \quad i=1,2, \\
\psi_{1}(-1)=\psi_{2}(1)=0,
\end{gathered}
$$


where $\sigma=2, \sigma_{s}=\frac{1}{2}, q=1, \mu_{1}=1 / \sqrt{3}, \mu_{2}=-1 / \sqrt{3}$, and $\omega_{1}=\omega_{2}=1.0$. It is easily seen that this problem has the following analytical solution:

$$
\begin{gathered}
\psi_{1}^{e}(x)=1-\exp (-2 \sqrt{3}(x+1)) \\
-\frac{\exp (-\sqrt{3}(x+1))}{(\sinh \sqrt{6}+\sqrt{2} \cosh \sqrt{6})}\left[\frac{\exp \left(\frac{\sqrt{6}}{2}(x-1)\right) \sinh \left[\left(\sqrt{3}+\frac{\sqrt{6}}{2}\right)(x+1)\right]}{1+\sqrt{2}}\right.
\end{gathered}
$$

$$
\left.+\frac{\exp \left(-\frac{\sqrt{6}}{2}(x-1)\right) \sinh \left[\left(\sqrt{3}-\frac{\sqrt{6}}{2}\right)(x+1)\right]}{\sqrt{2}-1}\right]
$$

$$
\psi_{2}^{e}(x)=1-\exp (-2 \sqrt{3}(1-x))
$$

$$
-\frac{\exp (-\sqrt{3}(1-x))}{\sinh \sqrt{6}+\sqrt{2} \cosh \sqrt{6}}\left[\frac{\exp \left(\frac{\sqrt{6}}{2}(x+1)\right) \sinh \left[\left(\sqrt{3}-\frac{\sqrt{6}}{2}\right)(1-x)\right]}{\sqrt{2}-1},\right.
$$

with the scalar flux $\phi^{e}(x)=\psi_{1}^{e}(x)+\psi_{2}^{e}(x)$, given by

$$
\phi^{e}(x)=2\left[1-\frac{\sqrt{2} \cosh \sqrt{6} x}{\sinh \sqrt{6}+\sqrt{2} \cosh \sqrt{6}}\right], \quad|x| \leqq 1 .
$$

Our numerical experiments will impose a sequence of nine uniform spatial meshes, with $h=2^{2-n}, 1 \leqq n \leqq 9$; and for each mesh generated, we obtain the corresponding approximate solution to (3.1) and (3.2). We refer the reader to [1], [5] for a discussion of the iteration procedure used to solve (3.1) and (3.2). As indicated in these works, each iteration consists of a sweep (a left-right-left sweep) through the space angle mesh to solve

$$
\begin{gathered}
\mu_{i} \frac{d}{d x} \psi_{i}^{(k+1)}(x)+\sigma \psi_{i}^{(k+1)}(x)=\sigma_{s} \sum_{m=1}^{2} \omega_{m} \psi_{m}^{(k)}(x)+q, \\
\quad|x| \leqq 1, \quad i=1,2, \quad k=0,1,2, \cdots, \\
\psi_{1}^{(k+1)}(-1)=\psi_{2}^{(k+1)}(1)=0 .
\end{gathered}
$$

The starting values for the iteration procedure were $\psi_{1}^{(0)}(x)=\psi_{2}^{(0)}(x)=0$. We terminate the iteration when the following relative error criterion between successive iterates is met:

$$
\max \left\{\left|\frac{\left[\mathbf{M}_{0}\left(\varphi^{(k+1)}-\varphi^{(k)}\right)\right]_{j}}{\left[\mathbf{M}_{0} \varphi^{(k+1)}\right]_{j}}\right|: j=1, \cdots, J\right\}<10^{-11}
$$

where $\varphi^{(k+1)}\left(\varphi^{(k)}\right)$ is the scalar flux associated with iteration $k+1(k)$ and equal to $\psi_{1}^{(k+1)}+\psi_{2}^{(k+1)}\left(\psi_{1}^{(k)}+\psi_{2}^{(k)}\right)$.

We give numerical results for the scalar flux only, since this is the dependent variable of primary interest in nuclear reactor calculations. The norm $\|\cdot\|_{e d}$ is defined 
by the left-hand side of (2.19), while the norm of a zeroth spatial Legendre moment vector associated with $f$ is

$$
\left\|\mathbf{M}_{0} f\right\|=\max \left\{\left|\left(\mathbf{M}_{0} f\right)_{j}\right|: j=1,2, \cdots, J\right\} .
$$

It should be recalled that a convergence rate is defined by $-\ln \left(E_{f} / E_{c}\right) / \ln 2$, when $E_{f}$ and $E_{c}$ are the errors associated with the finer and coarser meshes respectively. It can be seen that for this simple problem the asymptotic convergence rates derived theoretically for cell-edge and cell-average approximations are present on rather coarse meshes for the linear characteristic and quadratic continuous methods. The rates in Table 1 for $\left\|\mathbf{M}_{0}\left(\varphi-\varphi^{e}\right)\right\|$ for $n=9$ are explained by the fact that the orders of magnitude of the errors in the cell-average approximations were not decreased when computations were done for $n=9$. This occurrence is because of the finite precision arithmetic and of the criterion (3.5) stopping the iteration. To see a rate of order four for $\left\|\mathbf{M}_{0}\left(\varphi-\varphi^{e}\right)\right\|$ when $n=9$ for both methods, a stopping criterion of $10^{-13}$, say, should be used in (3.5) in conjunction with higher than double precision arithmetic.

TABLE 1

Convergence rates for cell-average and cell-edge scalar fluxes.

\begin{tabular}{l|l|c|c|c|c}
\hline & & \multicolumn{2}{|c|}{ Linear characteristic } & \multicolumn{2}{c}{ Quadratic continuous } \\
\cline { 3 - 6 }$n$ & $\varepsilon$ & $\left\|\varphi-\varphi^{e}\right\|_{\text {ed }}$ & $\left\|\mathbf{M}_{0}\left(\varphi-\varphi^{e}\right)\right\|$ & $\left\|\varphi-\varphi^{e}\right\|_{e d}$ & $\left\|\mathbf{M}_{0}\left(\varphi-\varphi^{e}\right)\right\|$ \\
\hline 1 & 6.93 & - & - & - & - \\
2 & 3.46 & .9122 & 4.8183 & 1.3802 & 5.0657 \\
3 & 1.73 & 4.0342 & .1782 & 4.2498 & .6179 \\
4 & 0.866 & 4.0231 & 3.0028 & 4.0942 & 3.0706 \\
5 & 0.433 & 4.0051 & 3.5302 & 4.0240 & 3.5489 \\
6 & 0.217 & 4.0012 & 3.7687 & 4.0060 & 3.7735 \\
7 & 0.108 & 4.0004 & 3.8849 & 4.0014 & 3.8862 \\
8 & 0.0541 & 4.0015 & 3.9423 & 3.9989 & 3.9432 \\
9 & 0.0271 & 4.0228 & -21.265 & 3.9774 & -21.265 \\
\hline
\end{tabular}

In Table 2 we compare the actual errors themselves for the cell-edge scalar fluxes generated by the linear characteristic and quadratic continuous methods. Our computations were done on an ITEL-AS/ 6 computer at Texas Tech University.

TABLE 2

Errors in cell-edge scalar fluxes.

\begin{tabular}{c|l|c|c}
\hline$n$ & $\varepsilon$ & Linear characteristic & Quadratic continuous \\
\hline 1 & 6.93 & $.51918(-1)$ & $.89151(-1)$ \\
2 & 3.46 & $.27587(-1)$ & $.34249(-1)$ \\
3 & 1.73 & $.16838(-2)$ & $.18002(-2)$ \\
4 & 0.866 & $.10356(-3)$ & $.10540(-3)$ \\
5 & 0.433 & $.64502(-5)$ & $.64789(-5)$ \\
6 & 0.217 & $.40279(-6)$ & $.40324(-6)$ \\
7 & 0.108 & $.25168(-7)$ & $.25178(-7)$ \\
8 & 0.0541 & $.15714(-8)$ & $.15748(-8)$ \\
9 & 0.0271 & $.96672(-10)$ & $.99975(-10)$ \\
\hline
\end{tabular}

As a final comment, we would like to point out that the quadratic continuous method can be made to be "positive" (in that nonnegative flux approximations can be computed from nonnegative data) when $\left|\varepsilon_{j i}\right|<6$, by utilizing the "negative source 
fix up" routine outlined in [1, p. 120] for the linear characteristic method. For this sample problem, positive values for $\psi_{i}\left(x_{j \pm 1 / 2}\right)$ and $\left[\mathbf{M}_{0} \psi\right]_{i, j}$ were obtained in each of the iterations for each mesh size.

Acknowledgments. The authors would like to express their appreciation to Dr. E. W. Larsen for suggesting the quadratic continuous method to the authors, and for his encouragement to this work generally.

\section{REFERENCES}

[1] R. E. Alcouffe, E. W. Larsen, W. F. Miller, JR. And B. R. Wienke, Computational efficiency of numerical methods for the multigroup discrete-ordinates neutron transport equation: The slab geometry case, Nucl. Sci. Eng., 71 (1979), pp. 111-127.

[2] P. M. ANSELONE, Collectively Compact Operator Approximation Theory, Prentice-Hall, Englewood Cliffs, NJ, 1971.

[3] P. BARBUCCI AND F. DiPAsQuANTONIO, Exponential supplementary equations for $\boldsymbol{S}_{n}$ methods: The one-dimensional case, Nucl. Sci. Eng., 63 (1977), pp. 179-187.

[4] D. V. Gopinath, A. NATARAJAN AND V. Sundararaman, Improved interpolation schemes in anisotropic source-flux iteration techniques, Nucl. Sci. Eng., 75 (1980), pp. 181-184.

[5] E. W. LARSEN AND W. F. Miller, JR., Convergence rates of spatial difference equations for the discrete-ordinates neutron transport equations in slab geometry, Nucl. Sci. Eng., 73 (1980), pp. 76-81.

[6] E. W. LARSEN AND P. NELSON, JR., Finite-difference approximations and superconvergence for the discrete-ordinate equations in slab geometry, this Journal, 19 (1982), pp. 334-348.

[7] SuResh M. Lee AND R. VAidyanathan, Comparison of the order of approximation in several spatial difference schemes for the discrete-ordinates transport equation in one-dimensional plane geometry, Nucl. Sci. Eng., 76 (1980), pp. 1-9.

[8] J. PITKÄRANTA, On the spatial differencing of the discrete ordinate neutron transport equation, this Journal, 15 (1978), pp. 859-869.

[9] R. VAIDYANATHAN, Effectiveness of a modified source interpolation scheme in discrete integral transport methods, Atomkernenergie, 29 (1977), pp. 301-302.

[10] —, A finite moments algorithm for particle transport problems, Nucl. Sci. Eng., 71 (1979), pp. 46-54. 